\begin{tabular}{|c|c|}
\hline Title & $\begin{array}{l}\text { Intramolecular Conjugate A ddition of a , } \beta \text { - Unsaturated L actones Having an A I kanenitrile Side Chain: } \\
\text { Stereocontrolled Construction of C arbocy cles with Quaternary Carbon A toms }\end{array}$ \\
\hline Author(s) & Y oshimura, Fumihiko; Torizuka, Makoto; Mori, Genki; Tanino, Keiji \\
\hline Citation & $\begin{array}{l}\text { Synlett, 2012(2), 251-254 } \\
\text { https://doi.org/10.1055/s 0031-1290074 }\end{array}$ \\
\hline Issue Date & 2012-01 \\
\hline Doc URL & http:/hdl.handle.net/2115/51017 \\
\hline Rights & (c) 2012 Georg Thieme V erlag \\
\hline Type & article (author version) \\
\hline File Information & Syn2012-2_251-254.pdf \\
\hline
\end{tabular}

Instructions for use 


\title{
Intramolecular Conjugate Addition of $\alpha, \beta$-Unsaturated Lactones Having an Alkaenenitrile Side Chain: Stereocontrolled Construction of Carbocycles with Quaternary Carbon Atoms
}

\author{
Fumihiko Yoshimura,* Makoto Torizuka, Genki Mori, Keiji Tanino* \\ Department of Chemistry, Faculty of Science, Hokkaido University, Sapporo 060-0810, Japan \\ Fax +81(11)706 4920; E-mail: fumi@ @sci.hokudai.ac.jp (F.Y.); ktanino@ sci.hokudai.ac.jp (K.T.)
}

\begin{abstract}
An efficient method for constructing carbocycles with all-carbon quaternary stereocenters has been developed on the basis of a stereoselective cyclization reaction of $\alpha, \beta$ unsaturated lactones having an alkanenitrile side chain. Treatment of the substrate with lithium hexamethyldisilazide (LiHMDS) in the presence of triisopropylsilyl chloride (TIPSCl) led to generation of the corresponding $\alpha$-cyano carbanion species which readily underwent an intramolecular conjugate addition reaction. It was found that the combined use of trimethylsilyl trifluoromethanesulfonate (TMSOTf) and triethylamine is also effective for the cyclization reaction without using a strong base. Interestingly, different stereochemical outcomes were observed in the two cyclization methods.
\end{abstract}

Key words: carbocycles, cyclization, Michael addition, nitriles, stereoselective synthesis

Development of an efficient method for construction of polysubstituted carbocycles in a stereoselective manner remains a considerable challenge in organic synthesis. In particular, carbocyclization reactions leading to formation of an all-carbon quaternary stereogenic center is very important in total synthesis of various natural products. ${ }^{1}$

Recently, we reported the asymmetric total synthesis of glycinoeclepin A on the basis of the cyclopentene annulation method for preparing key intermediate $\mathbf{2}$ (Scheme 1). ${ }^{2}$ The contiguous all-carbon quaternary stereogenic centers of bicyclic enone $\mathbf{2}$ were constructed by the highly stereoselective conjugate addition reaction using an $\alpha$-cyano carbanion species generated from nitrile $\mathbf{1}$.

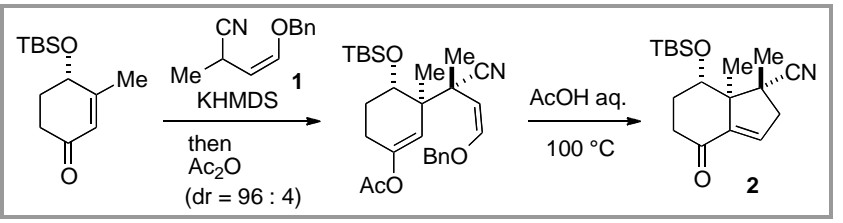

Scheme 1 The stereoselective intermolecular conjugate addition reaction of nitrile $\mathbf{1}$ under basic conditions

The result led us to investigate the intramolecular version of a conjugate addition reaction using an $\alpha$ cyano carbanion species (Scheme 2) ${ }^{3,4}$ With a view to inducing selective formation of an $\alpha$-cyano carbanion, $\alpha, \beta$-unsaturated lactone $\mathbf{3}$ possessing no enolizable proton was designed as the cyclization precursor.

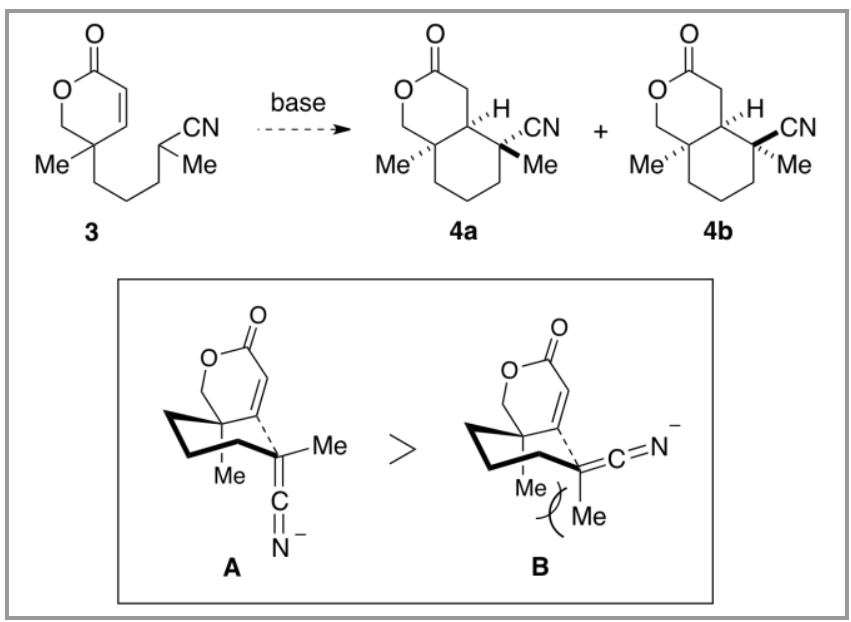

Scheme 2 An intramolecular conjugate addition reaction of an $\alpha, \beta$-unsaturated lactone having an alkanenitrile side chain

On treatment with a base, 3 would afford $\alpha$-cyano carbanion species which may undergo intramolecular conjugate addition reaction through transition state $\mathbf{A}$ or $\mathbf{B}$. We envisioned that formation of bicyclic compound 4a through transition state $\mathbf{A}$ may be preferred, because another transition state $\mathbf{B}$ leading to epimer $\mathbf{4 b}$ suffers from the steric repulsion between the two 1,3-diaxial methyl groups. In addition, the use of a cyano group, which is much less sterically demanding than an ester group, ${ }^{5}$ in substrate $\mathbf{3}$ would be effective for increasing the energy difference between the transition states $\mathbf{A}$ and $\mathbf{B}$.

At first, the cyclization precursor $\mathbf{3}$ was synthesized from commercially available ethyl tiglate (5) as shown in Scheme 3. Deconjugative $\alpha$-alkylation of 5 with 3chloro-1-iodopropane followed by three-step manipulation of the functional groups afforded primary alkyl iodide 7 . The nitrile moiety was introduced via a substitution reaction of 7 with the carbanion species generated from propionitrile. Construction of the lactone ring was achieved through conversion of THP ether $\mathbf{8}$ to the corresponding acrylate 9 followed by ring closing metathesis (RCM) catalyzed by Umicore M2. ${ }^{6}$ 


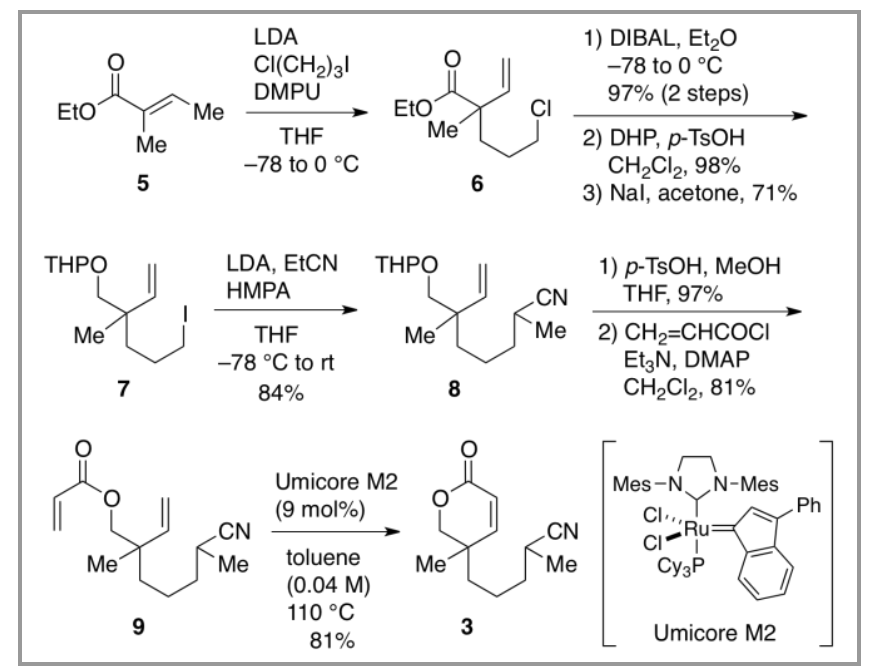

Scheme 3 Synthesis of lactone $\mathbf{3}$ having an alkanenitrile side chain

With unsaturated lactone $\mathbf{3}$ in hand, we investigated the cyclization reaction mediated by an amide base (Table 1). When 3 was treated with potassium hexamethyldisilazide (KHMDS) or sodium hexamethyldisilazide (NaHMDS) in THF, a complex mixture containing small amounts of bicyclic lactones $\mathbf{4 a}$ and $\mathbf{4} \mathbf{b}^{7}$ along with recovery of $\mathbf{3}$ was obtained (entries 1 and 2). On the other hand, the use of lithium hexamethyldisilazide (LiHMDS) led to a rather better result, and higher conversion of the reaction was observed without formation of any side-products (entry 3). While the combination of LiHMDS and hexamethylphosphoric triamide (HMPA) was found to enhance both yield and diastereomeric ratio of the products $(51 \%, \mathbf{4 a}: \mathbf{4 b}=86: 14)$, the condition also caused a considerable increase in the amount of the side-products (entry 4).

Table 1 Intramolecular conjudate addition reactions of lactone 3 under basic conditions ${ }^{\mathrm{a}}$

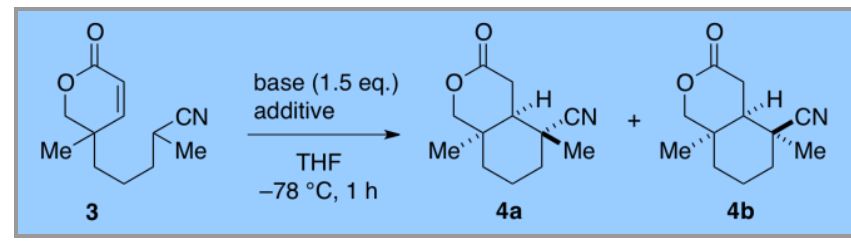

$\begin{array}{llllll}\text { entry } & \text { base } & \text { additive } & \text { yield }^{\mathrm{b}} & \mathbf{4 a} / \mathbf{4} \mathbf{b}^{\mathrm{b}} & \text { recovery }^{\mathrm{b}} \\ 1 & \text { KHMDS } & - & 11 \% & 1: 99 & 35 \% \\ 2 & \text { NaHMDS }- & 20 \% & 15: 85 & 25 \% \\ 3 & \text { LiHMDS }- & 29 \% & 50: 50 & 49 \% \\ 4 & \text { LiHMDS } & \text { HMPA } & 51 \% & 86: 14 & 7 \%\end{array}$

${ }^{\mathrm{a}}$ Typical reaction conditions: lactone $\mathbf{3}(0.2 \mathrm{mmol})$, base $(0.3$ mmol), additive $(1.0 \mathrm{mmol}), \operatorname{THF}(2 \mathrm{~mL}),-78^{\circ} \mathrm{C}$.

${ }^{\mathrm{b}}$ Determined by ${ }^{1} \mathrm{H}$ NMR.

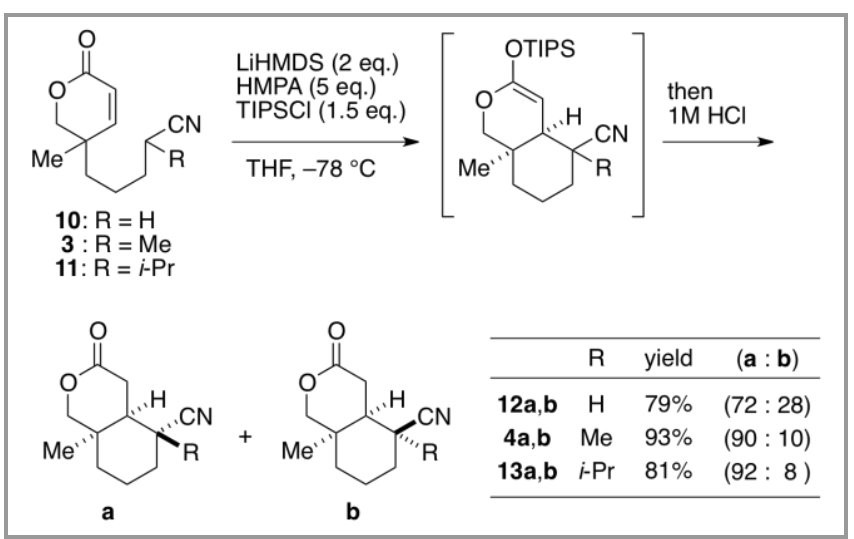

Scheme 4 Improved method for the intramolecular conjugate addition reactions of lactones $\mathbf{3}, \mathbf{1 0}$, and $\mathbf{1 1}$

These results suggested that the lactone enolate formed by the intramolecular conjugate addition reaction may undergo degradation or an intermolecular addition reaction. In order to exclude these possibilities, the reactions in the presence of a silylating reagent for trapping of the lactone enolate were examined. We were pleased to find that the use of triisopropylsilyl chloride (TIPSCl) gave good result as shown in Scheme $4 .^{8}$

Thus, upon treatment of $\mathbf{3}$ with LiHMDS (2 eq.) and TIPSCl (1.5 eq.) in the presence of HMPA (5 eq.) at $78{ }^{\circ} \mathrm{C}$, the intramolecular conjugate addition reaction proceeded smoothly to afford the cyclic ketene silyl acetal. Treatment of the reaction mixture with $1 \mathrm{M}$ $\mathrm{HCl}$ in one-pot gave a diastereomeric mixture of bicyclic lactones $\mathbf{4 a}$ and $\mathbf{4 b}$ in $93 \%$ isolated yield. ${ }^{9,10}$ Similarly, substrates $\mathbf{1 0}$ and $\mathbf{1 1},{ }^{11}$ possessing a different $\alpha$-substituent on the nitrile moiety, were transformed into the corresponding bicyclic lactones 12 and $\mathbf{1 3}$, respectively.

The steric effect of the $\alpha$-substituent plays an important role to control the diastereoselectivity in the cyclization reactions (Scheme 4). Thus, increasing the steric hindrance of the $\alpha$-substituent in the cyclization precursor $(\mathrm{H}<\mathrm{Me}<i$-Pr $)$ tends to increase the diastereomeric ratio of the product $(72: 28<90: 10<$ $92: 8)$. It is noteworthy that these stereochemical outcomes are consistent with that supposed from the transition state models A and $\mathbf{B}$ in Scheme 2.

During the course of the studies to find a suitable silylating reagent in the carbocyclization reaction, we realized that a similar transformation can be achieved without using a strong base such as LiHMDS (Scheme $5)$. Thus, when 3 was treated with TMSOTf (2 eq.) in the presence of $\mathrm{Et}_{3} \mathrm{~N}$ (4 eq.) in 1,2-dichloroethane at $50{ }^{\circ} \mathrm{C}$ for $1.5 \mathrm{~h}$, carbocyclization proceeded smoothly to afford $\mathbf{4 a}$ and $\mathbf{4 b}$ in $84 \%$ yield. ${ }^{12,13}$ Interestingly, this new method showed complementary steteochemical outcome on the newly formed quaternary stereogenic center as compared to LiHMDS/TIPSCl system; As a result, $\mathbf{4 b}$ was obtained 
as a major isomer in a 67:33 ratio to $4 \mathbf{a}$. The reaction mechanism of this cyclization would require further analysis; mechanistic investigations are currently ongoing in our laboratory. ${ }^{14}$

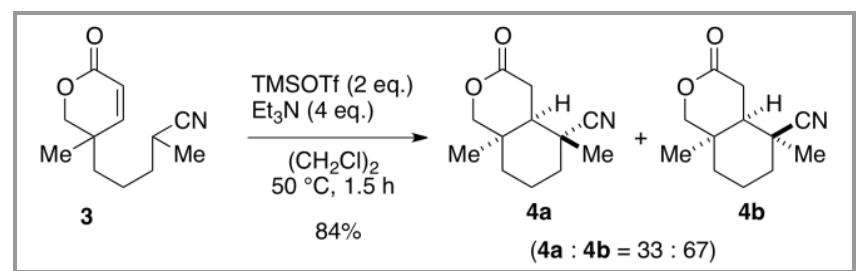

Scheme 5 Alternative method for carbocyclization of lactone 3

Having established the efficient carbocyclization procedures, the scope of these reactions was investigated by using several substrates as shown in Scheme 6. While LiHMDS/TIPSCl-mediated cyclization of the precursor $14^{11}$ having a $\gamma$-lactone moiety afforded the cis-fused cyclohexane derivative 15 in $94 \%$ yield, the diastereomeric ratio of $\mathbf{1 5}$ was lower than that of bicyclic lactone 4 . The cyclization of 14 by using TMSOTf/Et ${ }_{3} \mathrm{~N}$ system provided 15 in excellent yield with complementary stereochemistry on the newly formed quaternary stereogenic center similar to the case of $\mathbf{4}$. Formation of a five-membered ring was examined by the reaction of $16^{11}$ having a shorter side chain. Although the carbocyclization reaction proceeded smoothly in both procedures to afford 17, poor stereoselectivity was observed in these cases, suggesting that the rigid chair-like sixmembered transition state as depicted in Scheme 2 is essential in high stereoselectivity.

It is noteworthy that a base-sensitive substrate could be efficiently cyclized by the combination of TMSOTf and $\mathrm{Et}_{3} \mathrm{~N}$. For example, carbocyclization of coumarin derivative 18, which was readily synthesized from 2,6-dihydroxybenzaldehyde in three steps, ${ }^{11}$ proceeded smoothly to afford the tricyclic lactones $\mathbf{1 9}$ in $76 \%$ yield in a stereoselective manner (86:14). On the other hand, LiHMDS/TIPSCl-mediated cyclization of $\mathbf{1 8}$ did not give satisfactory results, where 19 was obtained only in $11 \%$ yield $(19 a: 19 b=50: 50)$.

To conclude, an efficient method for constructing carbocycles with all-carbon quaternary stereocenters has been developed on the basis of a stereoselective cyclization reaction of $\alpha, \beta$-unsaturated lactones having an alkanenitrile side chain. Treatment of the substrate with LiHMDS in the presence of TIPSCl and HMPA led to generation of the corresponding $\alpha$-cyano carbanion species which readily underwent an intramolecular conjugate addition reaction. It was found that the combined use of TMSOTf and $\mathrm{Et}_{3} \mathrm{~N}$ is also effective for the cyclization reaction without using a strong base. Interestingly, different stereochemical outcomes were observed in the two cyclization methods. Application of this methodology to natural product synthesis is in progress, and will be reported in due course.

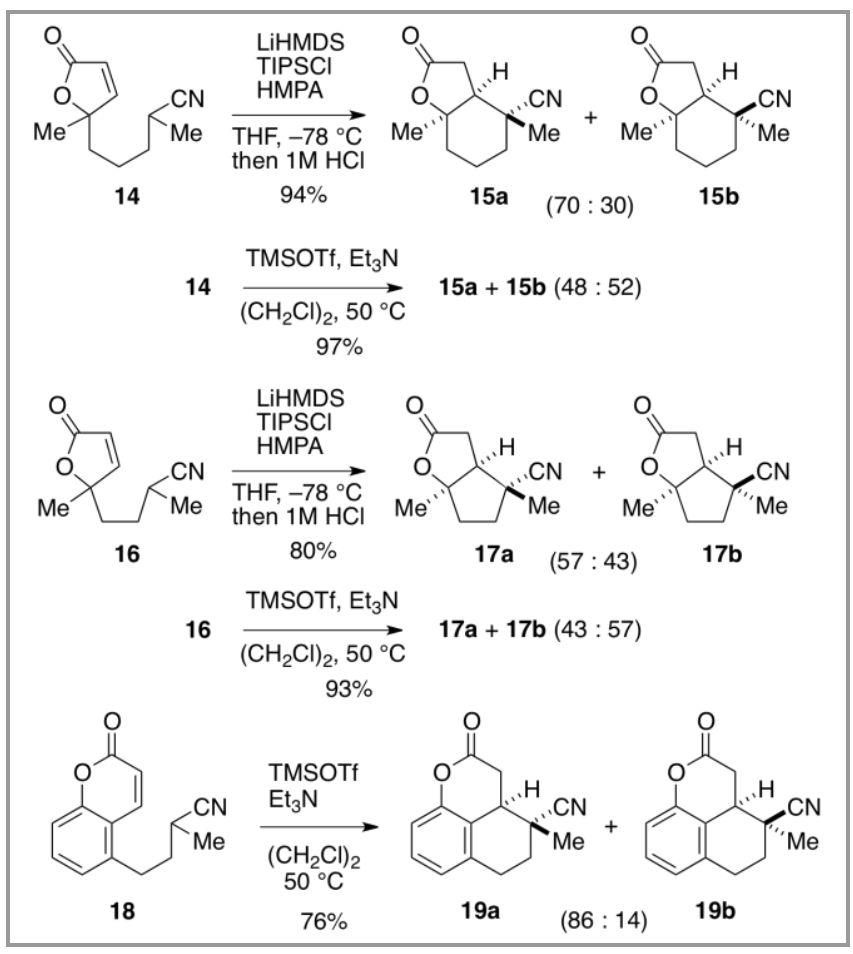

Scheme 6 Carbocyclization of various substrates

Supporting Information for this article is available online at http://www.thiemeconnect.com/ejournals/toc/synlett.

\section{Acknowledgement}

We acknowledge Dr. Eri Fukushi and Mr. Kenji Watanabe (GC-MS \& NMR Laboratory, Graduate School of Agriculture, Hokkaido University) for their mass spectral measurements. This work was supported by a Grant-in-Aid for Young Scientists (B) (No. 22710206) and the Global COE Program (Project No. B01: Catalysis as the Basis for Innovation in Materials Science) from the Ministry of Education, Culture, Sports, Science and Technology, Japan.

\section{References and Notes}

(1) For representative reviews of stereoselective construction of all-carbon quaternary stereogenic centers, see: (a) Christoffers, J.; Baro, A. Adv. Synth. Catal. 2005, 347, 1473-1482; (b) Denissova, I.; Barriault, L. Tetrahedron 2003, 59, 10105-10146; (c) Fuji, K. Chem. Rev. 1993, 93, 2037-2066.

(2) (a) Shiina, Y.; Tomata, Y.; Miyashita, M.; Tanino, K. Chem. Lett. 2010, 39, 835-837; (b) Tanino, K.; Tomata, Y.; Shiina, Y.; Miyashita, M. Eur. J. Org. Chem. 2006, 328-334.

(3) There are a few examples of the intramolecular conjugate addition reaction involving an $\alpha$-cyano carbanion intermediate. (a) Condon, S.; Ouarradi, A. E.; Métay, E.; Léonel, E.; Bourdonneau, M.; Nédélec, J.-Y. Tetrahedron 2008, 64, 9388-9395; (b) Shia, K.-S.; Jan, N.-W.; Zhu, J.-L.; Ly, T. W.; Liu, H.-L. Tetrahedron Lett. 1999, 40, 6753-6756. 
(4) The intramolecular alkylation reactions of nitriles provide useful carbocyclization method. For an excellent review of nitrile anion cyclization, see: Fleming, F. F.; Shook, B. C. Tetrahedron 2002, 58, 1-23.

(5) Eliel, E. L.; Wilen, S. H.; Mander, L. N. Stereochemistry of Organic Compounds; Wiley: New York, 1994, pp 696-697.

(6) Clavier, H.; Nolan, S. P. Chem. Eur. J. 2007, 13, 80298036.

(7) The configurations of $\mathbf{4 a}$ and $\mathbf{4 b}$ were unambiguously determined by ${ }^{1} \mathrm{H}$ NMR NOE experiments. See supporting information for details.

(8) The use of sterically demanding silylating reagent is of critical importance in this reaction. For example, the reaction in the presence of less bulky triethylsilyl chloride (TESCl) was accompanied by formation of the $\alpha$-silyl nitrile through trapping of the $\alpha$-cyano carbanion before cyclization.

(9) It should be noted that the use of a nitrile group was indispensable for the LiHMDS/TIPSCl-mediated cyclization. For example, when a nitrile group was replaced to an ester group, the reaction did not proceed efficiently; the bicyclic lactone was obtained only in $15 \%$ yield along with the recovery of the starting material in $63 \%$ yield.

$$
\text { (single isomer) }
$$

(10) General experimental procedure for carbocyclization by using LHMDS/TIPSCI system: To a cooled mixture of $\alpha, \beta$-unsaturated lactone 3 (32.4 mg, $0.156 \mathrm{mmol})$, TIPSCl (49.8 $\mu \mathrm{L}, 0.235 \mathrm{mmol})$, and HMPA $(136 \mu \mathrm{L}$, $0.782 \mathrm{mmol})$ in THF $(1.6 \mathrm{~mL})$ was slowly added a freshly prepared 1.0 M THF solution of LiHMDS (312 $\mu \mathrm{L}, 0.312 \mathrm{mmol}$ ) at $-78{ }^{\circ} \mathrm{C}$. After stirred at this temperature for $7 \mathrm{~h}$, the reaction was quenched with $1 \mathrm{M}$ $\mathrm{HCl}$ (aq.) (1.6 mL). The mixture was stirred at room temperature for $1.5 \mathrm{~h}$ and then diluted with EtOAc. After the layers were separated, the aqueous layer was extracted with EtOAc. The combined organic layers were washed successively with water and brine, dried over $\mathrm{MgSO}_{4}$, and concentrated under reduced pressure. The residue was purified by flash column chromatography on silica gel (hexane/EtOAc=5:1 1:1) to afford $\mathbf{4 a}(27.3 \mathrm{mg}, 84 \%)$ and $\mathbf{4 b}(2.9 \mathrm{mg}, 9 \%)$.

(11) See supporting information for the synthesis.

(12) Controlled experiments showed that neither TMSOTf nor $\mathrm{Et}_{3} \mathrm{~N}$ alone promoted this carbocyclization.

(13) General experimental procedure for carbocyclization by using TMSOTf/Et $\mathbf{t}_{3} \mathbf{N}$ system: To a mixture of $\alpha, \beta$ unsaturated lactone $\mathbf{3}(18.5 \mathrm{mg}, 89.0 \mu \mathrm{mol})$ and $\mathrm{Et}_{3} \mathrm{~N}$ $(50.2 \mu \mathrm{L}, 0.357 \mathrm{mmol})$ in 1,2 -dichloroethane $(0.45 \mathrm{~mL})$ was added TMSOTf $(32.3 \mu \mathrm{L}, 0.179 \mathrm{mmol})$ at room temperature. After stirred at $50{ }^{\circ} \mathrm{C}$ for $1.5 \mathrm{~h}$, the mixture was cooled to room temperature and a saturated aqueous $\mathrm{NaHCO}_{3}$ solution was added. The layers were separated, and then the aqueous layer was extracted with EtOAc. The combined organic layers were dried over $\mathrm{MgSO}_{4}$ and concentrated under reduced pressure. The residue was purified by flash column chromatography on silica gel (hexane/EtOAc=5:1 1:1) to afford $\mathbf{4 a}(5.1 \mathrm{mg}, 28 \%)$ and $\mathbf{4 b}(10.3 \mathrm{mg}, 56 \%)$.

(14) We could not detect any silyl ketene imine intermediates in the cyclization of $\mathbf{3}$ by using TMSOTf/Et $\mathrm{TH}_{3} \mathrm{~N}$ system when monitoring the reaction by ${ }^{1} \mathrm{H}$ and ${ }^{13} \mathrm{C}$ NMR. In this connection, Denmark and Wilson have recently reported the Lewis base catalyzed intermolecular conjugate addition of silyl ketene imines to $\alpha, \beta$ unsaturated carbonyl compounds, see: Denmark, S. E.; Wilson, T. W. Synlett 2010, 1723-1728. 